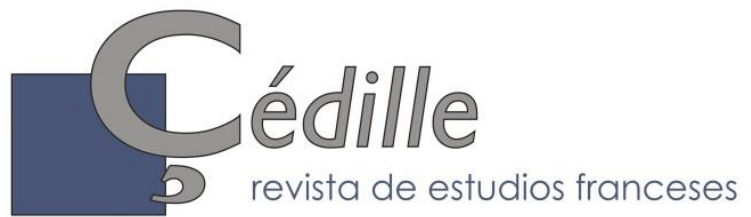

\title{
Salvaguarda de la lengua francesa en el mundo y de las lenguas minoritarias en Francia: políticas e instituciones
}

\author{
Isabel BRIALES BELLÓN \\ Universidad Pablo de Olavide \\ ibribel@upo.es
}

\section{Resumen}

Actualmente el número de hablantes de francés en el mundo asciende a 300 millones (Beck et al., 2018) y se encuentran repartidos en los cinco continentes. En este artículo analizamos los medios que existen para la expansión y preservación de la lengua francesa, siendo conscientes de que esta convive con lenguas hipercentrales, supercentrales, centrales y periféricas, algunas de ellas en peligro de extinción. Francofonía y diversidad van de la mano. Así, en el caso concreto de Francia, nos planteamos cuál será el futuro de la riqueza lingüística del país, tanto desde el punto de vista lingüístico como desde el político: ¿protección o desaparición?

Palabras clave: Francés. Organización Internacional de la Francofonía. Lengua minoritaria. Constitución. Protección.

Abstract

Nowadays, approximately 300 million people (Beck et al., 2018) in all five continents speak French. In this paper we analyse the current ways in which this language is expanded and preserved, keeping in mind that French coexists within hypercentral, supercentral, central and peripheral languages, some of them in danger of extinction. Francophonie and diversity walk together. We study the case of France and what the future of its linguistic wealth is, both from a linguistic and from a political perspective: protection or extinction?

Keywords: French. The International Organisation of La Francophonie. Minority language. Constitution. Protection.

Résumé

À ce jour, le nombre de francophones s'élève à 300 millions (Beck et al., 2018) et ils sont répartis sur les cinq continents. À travers cet article, il s'agit d'analys er les moyens existants pour l'expansion et la protection de la langue française, compte-tenu du fait qu'elle coexiste avec des langues hypercentrales, supercentrales, centrales et périphériques ; certaines d'entre-elles en danger d'extinction. Francophonie et diversité sont la main dans la main. Ainsi, dans le cas précis de la France, nous réfléchissons au futur de la richesse linguistique du

* Artículo recibido el 16/11/2018, aceptado el 16/05/2019. 
pays, non seulement du point de vu linguistique, mais aussi politique : protection ou disparition?

Mots clé : Français. Organisation international de la Francophonie. Langue minoritaire. Constitution. Protection.

\section{La lengua francesa en el mundo}

On parle désormais de francophonie avec un « $\mathrm{f}$ » minuscule pour désigner les locuteurs de français et de Francophonie avec un " $\mathrm{F}$ " majuscule pour figurer le dispositif institutionnel organisant les relations entre les pays francophones (Organisation Internationale de la Francophonie, 2018a).

De entre las organizaciones e instituciones que surgen en torno a la lengua francesa, destaca la Organisation Internationale de la Francophonie (en adelante OIF), que se creó en 1970 con el nombre de Agence de coopération culturelle et technique. La OIF está compuesta por 88 países que están unidos por el vínculo de la lengua y aúnan esfuerzos para progresar (OIF, 2018a). Estos países se dividen en estados miembros (61) y observadores (27) y se encuentran repartidos por todo el mundo (OIF, 2018b). Sus jefes de estado y de gobierno se reúnen periódicamente para tomar decisiones políticas relacionadas con la francofonía y llevan a cabo labores de cooperación multilateral, gracias a las siguientes entidades:

- Agence universitaire de la Francophonie

- TV5Monde

- Association internationale des maires francophones

- Université Senghor d'Alexandrie

Conviene puntualizar que los objetivos de la OIF (2018a) están más dirigidos hacia el respeto de los derechos humanos, la garantía de la democracia o la educación en aquellos países en los que se habla la lengua francesa, que a proteger el idioma como tal. Dichos objetivos se resumen en:

- Instauración y desarrollo de la democracia.

- Prevención, gestión y solución de conflictos, defensa del Estado de derecho y de los derechos humanos.

- Aumento del diálogo entre culturas y civilizaciones.

- Acercamiento de los pueblos gracias al conocimiento mutuo.

- Refuerzo de la solidaridad a través de acciones de cooperación multilateral para impulsar el desarrollo de las economías.

- Promoción de la educación y de la formación. 
Y ayudan a cumplir la misión que se le ha encomendado a la organización, esto es (OIF, 2018a):

- Promocionar la lengua francesa y la diversidad cultural y lingüística.

- Promover la paz, la democracia y los derechos humanos.

- Apoyar la educación, la formación, la educación superior y la investigación.

- Impulsar la cooperación al servicio del desarrollo sostenible.

Con el fin de conocer datos reales como, por ejemplo, en qué países se habla francés y si en cada uno de ellos es la lengua oficial o cooficial, o si es el idioma utilizado en las escuelas, se creó, dentro de la OIF (2018b), el Observatoire de la langue française (en adelante OLF), cuyo responsable es Alexandre Wolff.

Según los datos que aporta el informe de 2018 elaborado por Beck, Marcoux, Richard y Wolff, en el que participan el OLF y el Observatoire démographique et statistique de l'espace francophone, hay 300 millones de francófonos en el mundo (Beck et al., 2018: 149), hecho este que sitúa al francés en el quinto puesto de la lista de lenguas más habladas del mundo, por detrás del chino mandarín, el inglés, el español y el árabe. Sin embargo, cabe destacar que el francés es, junto al inglés, el único presente en los cinco continentes (OIF, 2018a).

Por otro lado, el mismo OLF establece que los francófonos en el mundo estarían divididos como muestran los siguientes gráficos (OIF, 2018a):

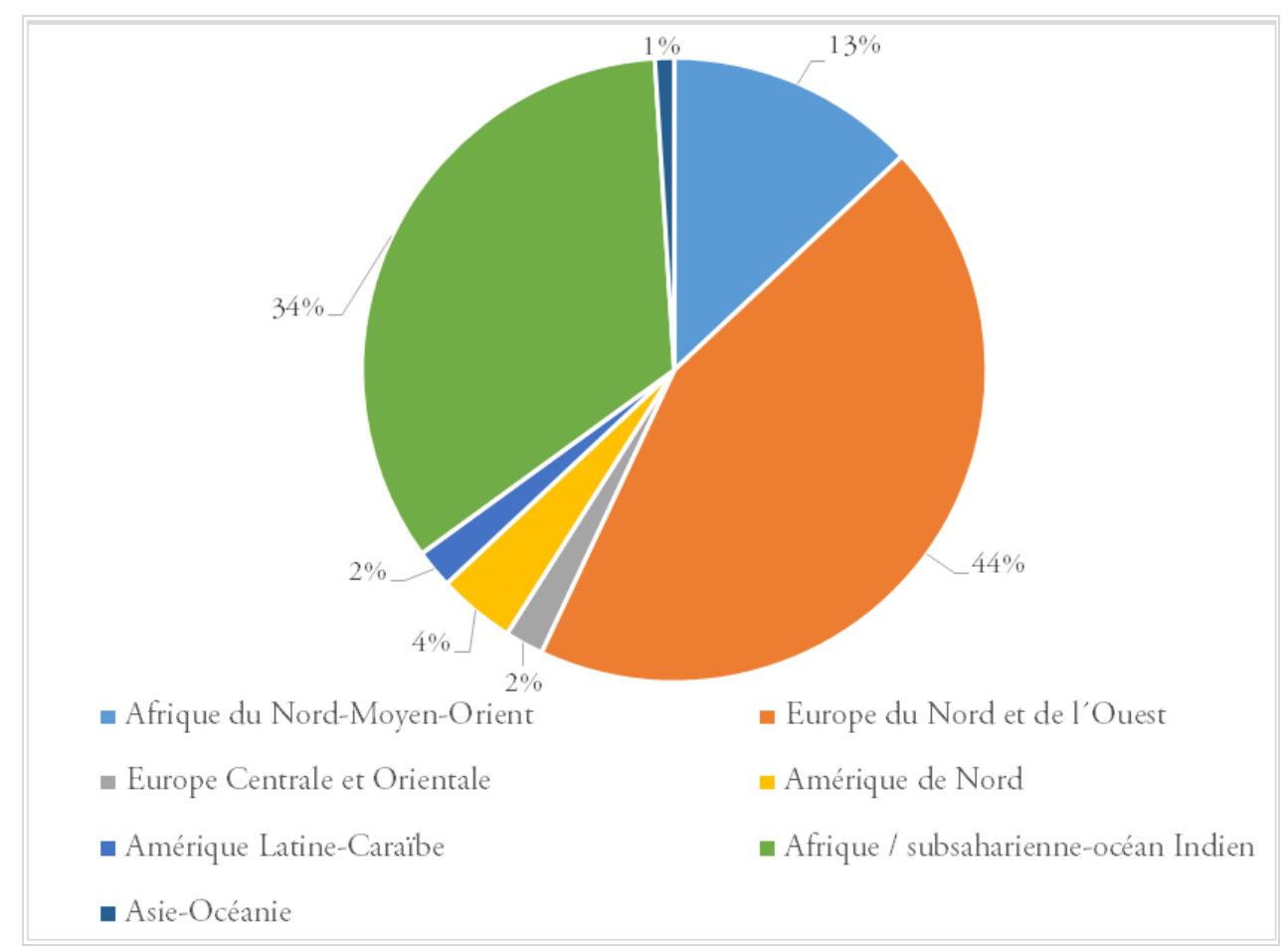

Figura 1. Francófonos en el mundo 
De los 300 millones de francófonos del mundo mencionados y que se recogen en la figura 1, 235 millones "viven en francés», es decir, la lengua que utilizan en la vida cotidiana es la francesa y estarían repartidos tal y como se muestra en la figura 2 :
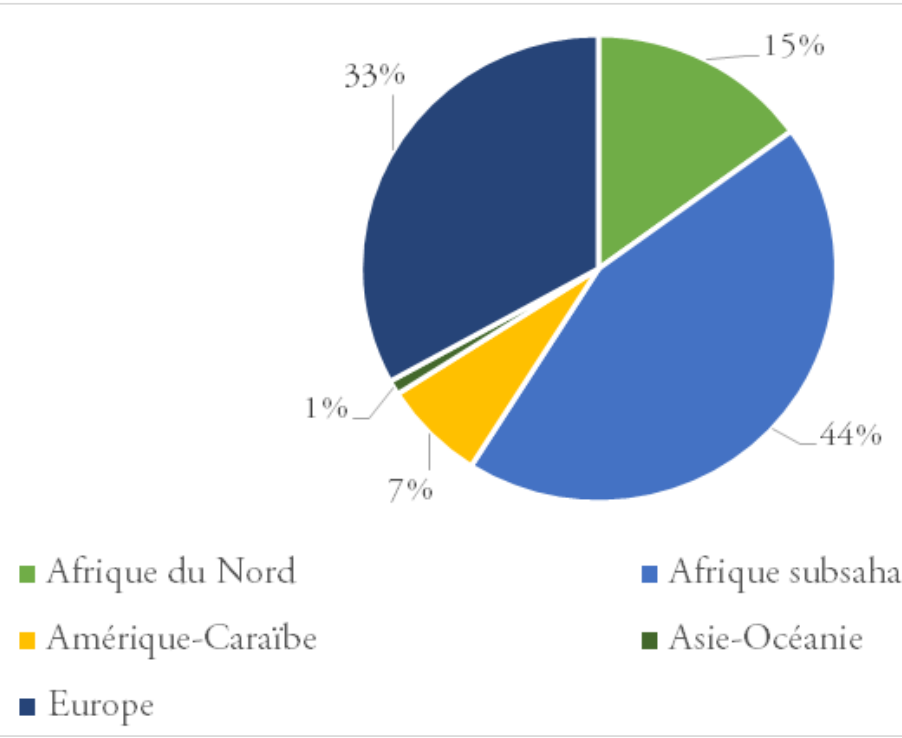

Figura 2. Hablantes habituales de francés en el mundo

De los datos que se desprenden de ambos gráficos, se deduce que Europa (Norte y Oeste) encabeza la lista según el número de hablantes de francés en valores absolutos, mientras que el África subsahariana y la parte oriental lideran el ranking si nos referimos a los francófonos que utilizan a diario la lengua francesa.

Además, el OLF enumera los países que tienen como única lengua oficial el francés, que serían: Benín, Burkina Faso, Congo, Costa de Marfil, Francia, Gabón, Guinea, Mali, Mónaco, Nigeria, República Democrática del Congo, Senegal y Togo (OIF, 2013).

En otros casos, el francés es lengua cooficial, al coexistir con otras lenguas, como, por ejemplo, en los siguientes países (OIF, 2013):

- Bélgica: francés, neerlandés y alemán

- Burundi: francés y kirundi

- Camerún: francés e inglés

- Canadá: francés e inglés

- Chad: francés y árabe

- Comoras: francés, comorense y árabe

- Guinea ecuatorial: francés, español y portugués

- Haití: francés y criollo

- Luxemburgo: francés, alemán y luxemburgués

- Madagascar: francés, inglés y malgache 
- República Centroafricana: francés y sango

- Ruanda: francés, inglés y kinyarwanda

- Seychelles: francés, inglés y criollo

- Suiza: francés, alemán, italiano y romanche

- Vanuatu: francés, inglés y bislama

- Yibuti: francés y árabe

A esta larga serie de países, hay que añadir 15 Estados federales o territorios autónomos más, en los que el francés es lengua oficial o cooficial (OIF, 2013), como es el caso, por ejemplo, de Puducherry, en la India, donde son lenguas oficiales el francés, junto al inglés, malayalam, tamil y télugu.

Así, Hagège (1987: 288) habla de una "patria francófona» que va más allá de los límites geográficos y políticos de cada país, una patria unida por la lengua francesa:

Cependant, l'attachement à la langue comme symbole de la patrie risque de constituer un obstacle dans l'entreprise de promotion de la francophonie. En effet, si la langue et patrie françaises peuvent être pensées solidairement par les Français, tel n'est pas le cas pour les autres pays francophones. C'est pourquoi la notion de patrie devrait prendre ici un contenu culturel et linguistique plutôt que politique. C'est d'une patrie francophone qu'il doit s'agir. Elle correspond non à une entité politique, mais à un vaste espace géographique et culturel, auquel participent tous les francophones du monde.

Gracias a los datos aportados por el OLF, es posible apreciar el alcance de la expansión de la lengua francesa en el mundo, en las dos vertientes que Calvet (1999: 248) recoge: la expansión concebida como el aumento del número de hablantes y la expansión entendida como el crecimiento del estatus de la lengua en un territorio (por ejemplo, adquisición del nivel de lengua oficial). Pero, ¿cuál es su futuro? El OLF estima que en 2050 el 85\% de los francófonos se encontrará en África, debido a los índices demográficos de la zona y al envejecimiento de la población de los países del hemisferio norte (OIF, 2013; Jacot, 2014). La probabilidad de que este pronóstico se cumpla aumenta debido a que son numerosos los países africanos que han implantado la lengua francesa como oficial en los centros educativos (OIF, 2013).

Más allá del futuro del francés en cifras y expansión, Calvet (2007: 153) se plantea su porvenir desde dos prismas diferentes: por un lado, el futuro formal del francés y, por otro, el efecto de las políticas lingüísticas.

Calvet (2007: 154) reflexiona sobre la existencia de una lengua hipercentral, el inglés, alrededor de la cual gravitan otras lenguas supercentrales muy importantes en cuanto a número de hablantes, como pueden ser el chino, el francés, el árabe o el 
español. A su vez, alrededor de estas giran entre 100 y 200 lenguas centrales, que son el eje de 4.000 o 5.000 lenguas periféricas. De esta forma, en cada nivel pueden producirse dos tipos de bilingüismo, es decir, un bilingüismo horizontal o adquisición de una lengua del mismo nivel, o un bilingüismo vertical o adquisición de una lengua de un nivel superior. Véase al respecto el siguiente cuadro:

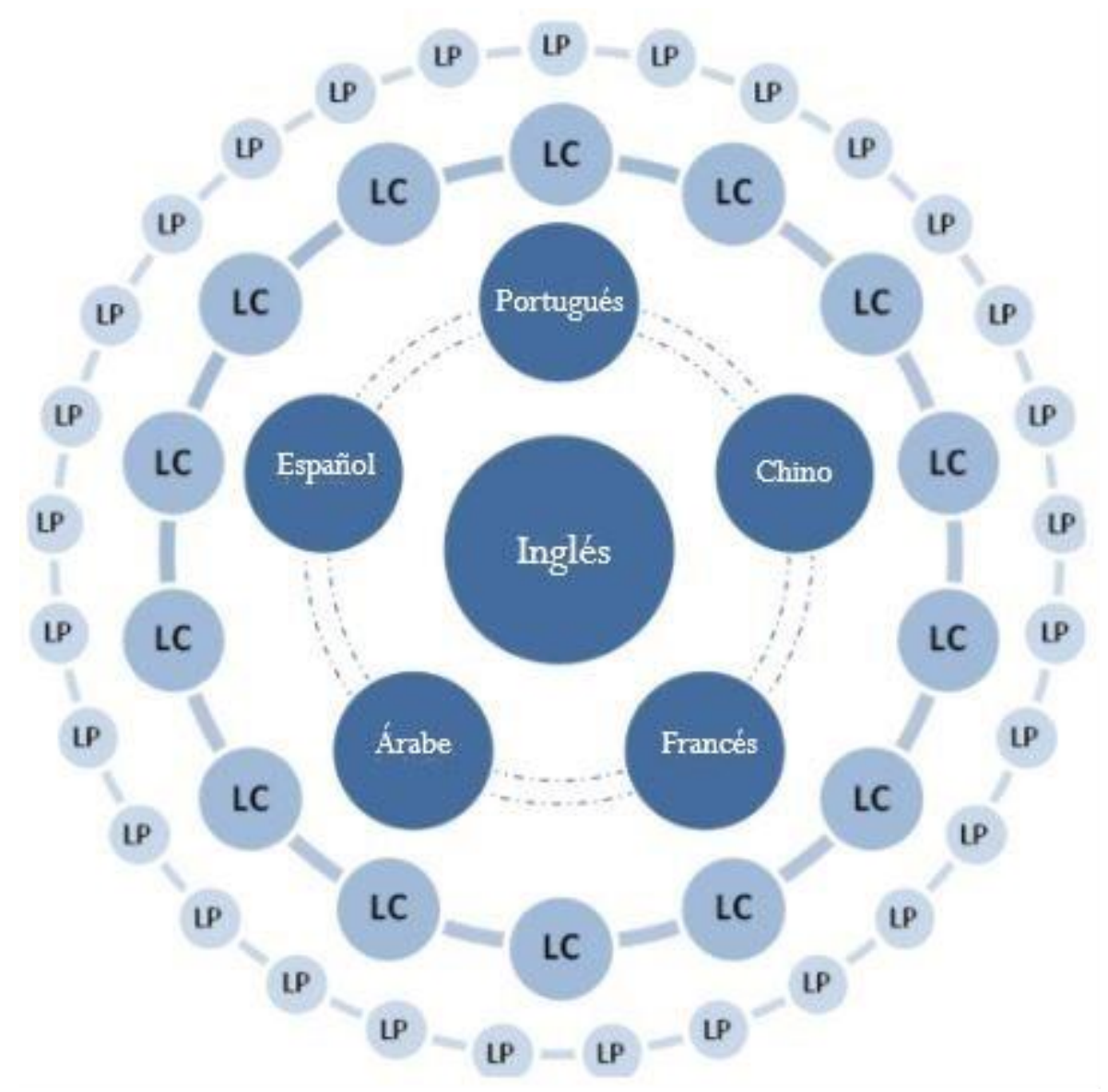

LC: Lenguas Centrales; LP: Lenguas Periféricas

Figura 3. Lenguas hipercentrales, supercentrales, centrales y periféricas

Habría que plantearse la relación que existe entre la posibilidad de que los hablantes sean bilingües y la clasificación de las lenguas en hipercentrales, supercentrales, centrales y periféricas. Calvet (2007: 154) asegura que en la mayor parte de los casos en los que se produce el bilingüismo vertical, este tiene lugar mediante el aprendizaje de una lengua de nivel superior, pues normalmente los padres transmiten a sus hijos la lengua de menor nivel, por ser la que se habla en el ámbito familiar, y es el individuo quien adquiere otra de nivel superior, por ser la que se practica en sociedad o en el ámbito laboral. Así, debido al contacto directo con otras lenguas habladas 
por los mismos interlocutores en otros ambientes y mediante la introducción de variantes, las lenguas se van extendiendo en cuanto a territorio y van modificando su vocabulario, gramática o fonética.

¿Se habla, por tanto, el mismo francés en Mali, Costa de Marfil, Canadá y Francia? Siendo consciente de que la respuesta es negativa, Calvet (2007: 157) se pregunta cuál es la directriz marcada por la Francofonía para el futuro, o lo que es lo mismo, si tiene en cuenta la diversidad real existente o si opta por imponer un modelo único de lengua, entendido como un «francés estándar». La OIF (2018c) da respuesta a esta cuestión abogando por la diversidad, por la conservación de las lenguas y las culturas ligadas a ellas.

A mayor abundamiento, Calvet (2007: 159) afirma que la defensa de la diversidad está relacionada con la convivencia de las lenguas del mundo (diversidad externa), con la oposición a la tendencia hacia una lengua única, ya que la eliminación de lenguas podría significar la anulación de identidades y pensamiento independiente, con la diversidad dentro de una misma lengua (diversidad interna) y con la defensa de los «colores» locales que adopta cada lengua según la zona en la que se hable, pues aún se pretende la unificación y centralización de las normas lingüísticas.

En este sentido, la francofonía debe reflexionar sobre la diversidad desde un punto de vista horizontal, vertical, interno y externo (Calvet, 2007: 160).

\section{Salvaguarda de la lengua francesa}

Tal y como hemos expuesto en el apartado anterior, existen organizaciones que velan por los intereses compartidos entre países en los que se habla francés, ya sea como lengua oficial o cooficial. De forma adicional, el gobierno francés ha creado un organismo interno, la Délégation générale à la langue française et aux langues de France (République Française, 2018a), que depende directamente del Ministerio de Cultura francés y, por tanto, tiene carácter oficial, con el fin de salvaguardar la lengua francesa, con objetivos puramente lingüísticos, y que dé respuesta a los problemas que puedan surgir en este ámbito.

La idea de fundar esta delegación nace en 1966, cuando el Primer Ministro francés George Pompidou crea el Haut comité pour la défense et l'expansion de la langue française. A partir de entonces, este organismo ha visto modificado tanto su nombre como su adscripción en diversas ocasiones. Desde 1996 depende del Ministerio de Cultura francés, adoptando en 2001 la denominación final de Délégation générale à la langue française et aux langues de France (en adelante DGLFLF).

Constituida como tal, sus prioridades son, además de difundir la lengua francesa en Europa y en el resto del mundo:

1. Velar por que los ciudadanos tengan acceso a la información en lengua francesa en situaciones de la vida cotidiana y se respete su dere- 
cho, recogido en la Constitución, a expresarse en esta misma lengua.

2. Fomentar el uso del francés como elemento de cohesión social a través de diversas iniciativas, entre las que destaca la colaboración de la DGLFLF en la creación del Diploma Inicial de Lengua Francesa, cuyo objetivo es facilitar la integración de los inmigrantes en la sociedad.

3. Enriquecer la lengua francesa y coordinar el trabajo de los actores que participan en la elaboración de los neologismos, como por ejemplo: logiciel en lugar de software, v.t.t. en vez de mountain bike o baladeur por walkman.

4. Impulsar la diversidad lingüística, potenciando el aprendizaje de lenguas extranjeras o de lenguas que se hablen en la misma familia.

5. Renovar las políticas de traducción, gracias al uso de las nuevas tecnologías o el desarrollo de la traducción automática.

6. Promocionar las lenguas de Francia en ámbitos relacionados con la cultura (teatro, libros, música, cine).

El foco de acción de esta delegación es Europa, por lo que el alcance es menor que el de la OIF. Sin embargo, es necesario tener presente que sus objetivos se centran en la lengua francesa, mientras que los de la OIF son más generales. Se podría decir que la DGLFLF vela por lo siguiente: «Tous les citoyens ont le droit démocratique, garanti par la loi, de recevoir une information et de s'exprimer dans leur langue (République Française, 2015b)».

Gilder (1993: 212), por su parte, insiste en que, si el objetivo es conservar el patrimonio lingüístico, el comienzo sería reforzar el conocimiento profundo de la lengua francesa de los hablantes nativos desde la escuela. Para ello propone, entre otras, las siguientes ideas: mejorar la formación lingüística de los profesores, saber transmitir la gramática de tal forma que entusiasme a los alumnos de todas las edades, invitar a escritores en las aulas, emplear una metodología lúdica a la hora de impartir clases de lengua, revivir las figuras retóricas y el gusto por la lectura, enseñar la historia de la lengua francesa, volver a recitar poemas y retransmitir cursos de francés por televisión.

\section{La Carta europea de las lenguas regionales o minoritarias}

Recordemos que la denominación de la delegación creada por el Gobierno de Francia para velar por la lengua francesa es Délégation générale à la langue française et aux langues de France. De ahí se deduce claramente que la realidad lingüística en Francia no es monolingüe, es decir, que el francés convive con otras lenguas, algunas de ellas minoritarias y en peligro de extinción. 
Conscientes de esta situación de convivencia, que no sólo se da con la lengua francesa y en Francia, el Consejo de Europa (Gobierno de España, 2015) adoptó como uno de sus objetivos principales proteger las lenguas europeas históricas regionales o minoritarias, ya que algunas de ellas están a punto de desaparecer, así como contribuir en el desarrollo de la riqueza cultural y las tradiciones en Europa, ayudando por tanto a que se garanticen principios como la igualdad, la democracia y la diversidad cultural.

Para velar por todo ello, el Consejo aprobó la Carta europea de las lenguas regionales o minoritarias (Conseil de l'Europe, 1992) en Estrasburgo el 5 de noviembre de 1992.

Abordamos este tema y nos basamos en la Carta europea de las lenguas regionales o minoritarias (Conseil de l'Europe, 1992) en nuestro estudio porque consideramos de gran valor que el Consejo de Europa, organización internacional que conlleva la implicación de numerosos países, se preocupe por la supervivencia de las lenguas minoritarias.

Cerquiglini, en 1999, en su informe sobre la aplicación de la Carta en Francia, recuerda que tanto esta como el documento explicativo adjunto, instan a todos los países miembros a dejar a un lado los resentimientos y la política, y a que apliquen las disposiciones de la Carta en favor de las lenguas maltratadas por la historia y, actualmente, por los medios de comunicación (Leclerc, 2017).

La Carta europea de las lenguas regionales o minoritarias nació con los objetivos y principios que a continuación se detallan:

1. En materia de lenguas regionales o minoritarias, las partes basarán su política, legislación y práctica en los siguientes objetivos y principios:

a. Reconocer las lenguas regionales o minoritarias como expresión de riqueza cultural.

b. Respetar el área geográfica de cada lengua regional o minoritaria.

c. Llevar a cabo actividades de promoción de las lenguas regionales o minoritarias con el fin de protegerlas.

d. Impulsar y favorecer el uso oral y escrito de las lenguas regionales o minoritarias tanto en la vida pública como privada.

e. Mantener y potenciar las relaciones, en los ámbitos que figuran en la Carta, entre los grupos que practican una lengua regional o minoritaria y otros grupos que en el mismo Estado hablen una lengua de forma idéntica o parecida; además, impulsar las relaciones de tipo cultural entre grupos de un Estado que hablen lenguas diferentes.

f. Propiciar las formas y medios adecuados para la enseñanza y el aprendizaje de las lenguas regionales o minoritarias en todos los niveles. 
g. Facilitar los medios para que las personas que vivan en una zona donde se hable una lengua regional o minoritaria que no conocen, puedan aprenderla si les interesa.

h. Promocionar la investigación y el estudio de las lenguas regionales o minoritarias en universidades o instituciones equivalentes.

i. Promocionar formas adecuadas de intercambios transnacionales, en los ámbitos que figuran en la Carta, para las lenguas regionales o minoritarias practicadas de manera similar o idéntica en dos o más Estados.

2. Las partes se comprometen a:

a. Eliminar, si aún no lo han hecho, cualquier distinción, exclusión, restricción o preferencia injustificada relacionada con la práctica de una lengua regional o minoritaria y que tenga como finalidad desmotivar o poner en peligro su mantenimiento o desarrollo. La adopción de medidas especiales para favorecer las lenguas regionales o minoritarias, con el objetivo de promover la igualdad entre los hablantes de estas lenguas y el resto de la población o que tengan en cuenta su situación particular, no serán consideradas como un acto de discriminación hacia los hablantes de lenguas mayoritarias.

b. Promover, con las medidas necesarias, la comprensión mutua entre todos los grupos lingüísticos del país, incluyendo el respeto, la comprensión y la tolerancia hacia las lenguas regionales o minoritarias en los objetivos en educación y formación en el país, así como a motivar a los medios de comunicación para que persigan este mismo objetivo.

c. Tener en cuenta las necesidades y deseos de los hablantes de estas lenguas. Se comprometen a crear, si fuera necesario, órganos de asesoramiento a las autoridades sobre cuestiones relacionadas con las lenguas regionales o minoritarias.

d. Aplicar, mutatis mutandis, los principios enumerados en los puntos 1 a 4 a las lenguas no territoriales. La naturaleza y el ámbito de las medidas aplicadas a estas lenguas se determinarán de manera flexible, teniendo en cuenta las necesidades y los deseos de sus hablantes y respetando las tradiciones y características de los grupos.

Como se ha mencionado anteriormente, estos objetivos y principios deben aplicarse a las lenguas regionales o minoritarias habladas en los países firmantes. Para comprender mejor a qué lenguas afectan las disposiciones recogidas en la Carta, es necesario tener claro el significado de 'lengua regional o minoritaria', de 'territorio en el que una lengua regional o minoritaria se practica' y de 'lenguas no territoriales', 
conceptos todos ellos que se definen en el artículo 1 de la Carta de la siguiente manera:

a) par l'expression «langues régionales ou minoritaires», on entend les langues:

i. pratiquées traditionnellement sur un territoire d'un État par des ressortissants de cet État qui constituent un groupe numériquement inférieur au reste de la population de l'État; et

ii. différentes de la (des) langue(s) officielle(s) de cet État; elle n'inclut ni les dialectes de la (des) langue(s) officielle(s) de l'État ni les langues des migrants;

b) par «territoire dans lequel une langue régionale ou minoritaire est pratiquée», on entend l'aire géographique dans laquelle cette langue est le mode d'expression d'un nombre de personnes justifiant l'adoption des différentes mesures de protection et de promotion prévues par la présente Charte;

c) par «langues dépourvues de territoire», on entend les langues pratiquées par des ressortissants de l'État qui sont différentes de la (des) langue(s) pratiquée(s) par le reste de la population de l'État, mais qui, bien que traditionnellement pratiquées sur le territoire de l'État, ne peuvent pas être rattachées à une aire géographique particulière de celui-ci (Conseil de l'Europe, 1992).

Una vez aclarados suficientemente estos conceptos y partiendo de los principios y objetivos de la Carta, los países firmantes se comprometen a aplicar al menos treinta y cinco apartados o subapartados de los siete artículos que se recogen en la parte III de la Carta, donde se especifican las medidas que se deben adoptar para impulsar el uso de las lenguas regionales o minoritarias en la vida pública. La parte III de la Carta está compuesta por los artículos numerados del 8 al 14, que abarcan los ámbitos que a continuación se detallan:

- Artículo 8. Educación

- Artículo 9. Justicia

- Artículo 10. Autoridades administrativas y servicios públicos

- Artículo 11. Medios de comunicación

- Artículo 12. Material y actividades culturales

- Artículo 13. Vida económica y social

- Artículo 14. Intercambios transfronterizos

Cada país firmante de la Carta está obligado a incluir tres apartados o subapartados del artículo 8 (Educación) y tres del 12 (Material y actividades culturales), así como uno del artículo 9 (Justicia), uno del 10 (Autoridades administrativas y servicios públicos), uno del 11 (Medios de comunicación) y uno del 13 (Vida económica y social). El siguiente paso para poner en práctica las disposiciones seleccionadas de entre los artículos mencionados, es que cada Estado firmante especifique a 
qué lengua minoritaria, regional u oficial, cuyo uso esté menos extendido en una parte o en todo su territorio, aplica los artículos elegidos.

De los 47 países miembros que conforman el Consejo de Europa, 25 han ratificado la Carta europea de las lenguas regionales o minoritarias, mientras que parte de los que no lo han hecho, 8 en total, aluden a la menor importancia que este tema tiene en su territorio.

A continuación, recogemos el cuadroque describe la situación de cada uno de los países que forman parte del Consejo de Europa con respecto a la Carta europea de las lenguas regionales o minoritarias (Conseil de l'Europe, 2018) ${ }^{1}$ :

Traité ouvert à la signature des États membres et à l'adhésion des États non membres. Situation au 10/11/20182

\begin{tabular}{|l|c|c|c|c|c|c|c|c|c|c|}
\cline { 2 - 10 } \multicolumn{1}{c|}{} & Signature & Ratification & $\begin{array}{c}\text { Entrée EN } \\
\text { VIGueur }\end{array}$ & Renv & R & D & A & T & C & O \\
\hline Albanie & & & & & & & & & & \\
\hline Allemagne & $5 / 11 / 1992$ & $16 / 9 / 1998$ & $1 / 1 / 1999$ & & & $\mathrm{X}$ & & & & \\
\hline Andorre & & & & & & & & & & \\
\hline Arménie & $11 / 5 / 2001$ & $25 / 1 / 2002$ & $1 / 5 / 2002$ & & & $\mathrm{X}$ & & & & \\
\hline Autriche & $5 / 11 / 1992$ & $28 / 6 / 2001$ & $1 / 10 / 2001$ & & & $\mathrm{X}$ & & & & \\
\hline Azerbaïdjan & $21 / 12 / 2001$ & & & & & $\mathrm{X}$ & & & & \\
\hline Belgique & & & & & & & & & & \\
\hline Bosnie-Herzégovine & $7 / 9 / 2005$ & $21 / 9 / 2010$ & $1 / 1 / 2011$ & & & $\mathrm{X}$ & & & & \\
\hline Bulgarie & & & & & & & & & & \\
\hline Chypre & $12 / 11 / 1992$ & $26 / 8 / 2002$ & $1 / 12 / 2002$ & & & $\mathrm{X}$ & & & & \\
\hline Croatie & $5 / 11 / 1997$ & $5 / 11 / 1997$ & $1 / 3 / 1998$ & & $\mathrm{X}$ & $\mathrm{X}$ & & & & \\
\hline Danemark & $5 / 11 / 1992$ & $8 / 9 / 2000$ & $1 / 1 / 2001$ & & & $\mathrm{X}$ & & & $\mathrm{X}$ & \\
\hline Espagne & $5 / 11 / 1992$ & $9 / 4 / 2001$ & $1 / 8 / 2001$ & & & $\mathrm{X}$ & & & & \\
\hline Estonie & & & & & & & & & & \\
\hline Finlande & $5 / 11 / 1992$ & $9 / 11 / 1994$ & $1 / 3 / 1998$ & & & $\mathrm{X}$ & & & & \\
\hline
\end{tabular}

${ }^{1}$ Este cuadro recoge las fechas en las que se produjeron la adhesión, la ratificación y la entrada en vigor de la Carta-si procede- en los 47 países que forman parte del Consejo de Europa, así como las particularidades de algunos de ellos, como puede ser la adhesión decidida por referendum.

${ }^{2}$ Renvois :

(56) Dates de signature et de ratification par l'union d'état de Serbie-Monténégro.

A. Adhésion - S. Signature sans réserve de ratification - SU. Succession - R. signature ad referendum. R. Réserves - D. Déclarations - A. Autorités - T. Application territoriale - C. Communication - O. Objection. 


\begin{tabular}{|c|c|c|c|c|c|c|c|c|c|c|}
\hline & SIGNATURE & RATIFICATION & $\begin{array}{c}\text { ENTRÉE EN } \\
\text { VIGUEUR }\end{array}$ & RENV & $\mathrm{R}$ & $\mathrm{D}$ & A & $\mathrm{T}$ & C & $\mathrm{O}$ \\
\hline France & 7/5/1999 & & & & & $\mathrm{X}$ & & & & \\
\hline \multicolumn{11}{|l|}{ Géorgie } \\
\hline \multicolumn{11}{|l|}{ Grèce } \\
\hline Hongrie & $5 / 11 / 1992$ & $26 / 4 / 1995$ & $1 / 3 / 1998$ & & & $\mathrm{X}$ & & & & \\
\hline \multicolumn{11}{|l|}{ Irlande } \\
\hline Islande & 7/5/1999 & & & & & & & & & \\
\hline Italie & $27 / 6 / 2000$ & & & & & & & & & \\
\hline \multicolumn{11}{|l|}{ Lettonie } \\
\hline Liechtenstein & $5 / 11 / 1992$ & 18/11/1997 & $1 / 3 / 1998$ & & & $\mathrm{X}$ & & & & \\
\hline \multicolumn{11}{|l|}{ Lituanie } \\
\hline Luxembourg & $5 / 11 / 1992$ & $22 / 6 / 2005$ & $1 / 10 / 2005$ & & & & & & & \\
\hline Macédoine (ex- Youg) & 25/7/1996 & & & & & & & & & \\
\hline Malte & $5 / 11 / 1992$ & & & & & & & & & \\
\hline Moldova & $11 / 7 / 2002$ & & & & & & & & & \\
\hline \multicolumn{11}{|l|}{ Monaco } \\
\hline Monténégro & $22 / 3 / 2005$ & $15 / 2 / 2006$ & $6 / 6 / 2006$ & 56 & & & & & & \\
\hline Norvège & 5/11/1992 & $10 / 11 / 1993$ & $1 / 3 / 1998$ & & & $\mathrm{X}$ & & & & \\
\hline Pays-Bas & $5 / 11 / 1992$ & $2 / 5 / 1996$ & $1 / 3 / 1998$ & & & $\mathrm{X}$ & & $\mathrm{X}$ & & \\
\hline Pologne & $12 / 5 / 2003$ & $12 / 2 / 2009$ & $1 / 6 / 2009$ & & & $\mathrm{X}$ & & & & \\
\hline \multicolumn{11}{|l|}{ Portugal } \\
\hline République tchèque & $9 / 11 / 2000$ & $15 / 11 / 2006$ & $1 / 3 / 2007$ & & & $\mathrm{X}$ & & & & \\
\hline Roumanie & $17 / 7 / 1995$ & $29 / 1 / 2008$ & $1 / 5 / 2008$ & & & $\mathrm{X}$ & & & & \\
\hline Royaume-Uni & $2 / 3 / 2000$ & $27 / 3 / 2001$ & $1 / 7 / 2001$ & & & $\mathrm{X}$ & & $\mathrm{X}$ & & \\
\hline Russie & $10 / 5 / 2001$ & & & & & & & & & \\
\hline \multicolumn{11}{|l|}{ Saint-Marin } \\
\hline Serbie & $22 / 3 / 2005$ & $15 / 2 / 2006$ & $1 / 6 / 2006$ & 56 & & $\mathrm{X}$ & & & & \\
\hline Slovaquie & $20 / 2 / 2001$ & $5 / 9 / 2001$ & $1 / 1 / 2002$ & & & $\mathrm{X}$ & & & & \\
\hline Slovénie & 3/7/1997 & $4 / 10 / 2000$ & $1 / 1 / 2001$ & & & $\mathrm{X}$ & & & & \\
\hline Suède & $9 / 2 / 2000$ & $9 / 2 / 2000$ & $1 / 6 / 2000$ & & & $\mathrm{X}$ & & & & \\
\hline Suisse & 8/10/1993 & $23 / 12 / 1997$ & 1/4/1998 & & & $\mathrm{X}$ & & & & \\
\hline Turquie & & & & & & & & & & \\
\hline
\end{tabular}




\begin{tabular}{|c|c|c|c|c|c|c|c|c|c|c|}
\hline & SigNATURE & RATIFICATION & $\begin{array}{l}\text { ENTRÉE EN } \\
\text { VIGUEUR }\end{array}$ & RENV & $\mathrm{R}$ & $\mathrm{D}$ & A & $\mathrm{T}$ & $\mathrm{C}$ & $\mathrm{O}$ \\
\hline Ukraine & 2/5/1996 & $19 / 9 / 2005$ & $1 / 1 / 2006$ & & & $\mathrm{X}$ & & & & \\
\hline & \multicolumn{4}{|c|}{ Nombre total de signatures non suivies de ratifications } & 8 & & & & & \\
\hline & \multicolumn{4}{|c|}{ Nombre total de ratifications/adhésions } & 25 & & & & & \\
\hline
\end{tabular}

Tabla 1. Firma y ratificación de la Carta europea de las lenguas regionales o minoritarias.

De los datos expuestos en la Tabla 1, llama la atención que Francia, país en el que conviven gran variedad de lenguas regionales, no haya finalizado el proceso y ratificado el documento. Esto se debe a que en la Constitución francesa (Conseil Constitutionnel de la République Française, 2018) se especifica que la lengua oficial es el francés, tal y como podemos comprobar en el siguiente párrafo, por lo que no hay cabida para el reconocimiento de las lenguas regionales que se propone en la Carta:

ARTICLE 2.

La langue de la République est le français.

L'emblème national est le drapeau tricolore, bleu, blanc, rouge.

L'hymne national est La Marseillaise.

La devise de la République est Liberté, Égalité, Fraternité.

Son principe est : gouvernement du peuple, par le peuple et pour le peuple.

De todas formas, Cerquiglini (apud Leclerc, 2017) aclara que la firma de la Carta está vinculada al reconocimiento de nueve objetivos y principios generales para todas las lenguas regionales o minoritarias del país, mientras que la ratificación implica la aplicación de los apartados seleccionados de la parte III de la Carta en las lenguas regionales o minoritarias elegidas.

La única solución para ratificar el documento y finalizar el proceso pasa por modificar la Constitución. De hecho, este punto conflictivo se incluyó en el programa del expresidente Hollande para ganar las elecciones de 2012 (Barbière, 2014).

Posteriormente, la Asamblea nacional francesa redactó, en enero de 2014, un artículo con el propósito de modificar la Constitución y hacerla compatible con la Carta, especificando que los hablantes de lenguas minoritarias no adquieren derechos suplementarios tras la ratificación y en qué situaciones es obligatorio el uso de la lengua francesa (Bartolone, 2014):

ARTICLE UNIQUE

Après l'article 53-2 de la Constitution, il est inséré un article 53-3 ainsi rédigé :

«Art. 53-3. - La République peut ratifier la Charte européenne des langues régionales ou minoritaires adoptée à Strasbourg le 
5 novembre 1992, signée le 7 mai 1999, complétée par la déclaration interprétative exposant que :

"1. L'emploi du terme de «groupes» de locuteurs dans la partie II de la charte ne conférant pas de droits collectifs pour les locuteurs des langues régionales ou minoritaires, le Gouvernement de la République interprète la charte dans un sens compatible avec la Constitution, qui assure l'égalité de tous les citoyens sans distinction d'origine, de race ou de religion ;

«2. Le $d$ du 1 de l'article 7 et les articles 9 et 10 de la charte posent un principe général n'allant pas à l'encontre de l'article 2 de la Constitution, en application duquel l'usage du français s'impose aux personnes morales de droit public et aux personnes de droit privé dans l'exercice d'une mission de service public, ainsi qu'aux usagers dans leurs relations avec les administrations et services publics ».

Délibéré en séance publique, à Paris, le 28 janvier 2014.

Así, llegado el momento, la Asamblea Nacional francesa tendría que especificar a qué lenguas dentro de su competencia afectaría la Carta europea de las lenguas regionales o minoritarias (Urvoas, 2014). Por el momento, no existe una lista cerrada, pero proponen una aproximación, gracias a la información que facilita la DGLFLF sobre las lenguas regionales o minoritarias habladas en Francia o en zonas de su competencia:

Lors de la signature de la Charte, la France a déclaré qu'elle indiquerait dans son instrument de ratification les langues régionales auxquelles les engagements qui précèdent, prévus dans la partie III, s'appliqueraient.

À l'heure actuelle, il n'est donc pas possible de disposer d'une liste exhaustive des langues qui seraient effectivement concernées: il appartiendra au pouvoir exécutif de dresser cette liste lors de la ratification de la Charte. Pour autant, il est loisible de se reporter, à titre indicatif, au panorama des langues régionales dressé par la Délégation générale à la langue française et aux langues de France, qui relève du ministère de la Culture et de la communication (voir l'encadré ci-après) (35).

Rappelons par ailleurs que les objectifs et principes prévus dans la partie II (article 7) s'appliquent à l'ensemble des langues satisfaisant aux définitions de la Charte, indépendamment de la liste établie par chaque État (36).

Les langues régionales en France aujourd'hui :

France métropolitaine:

Basque, breton, catalan, corse, dialectes alémanique et francique (alsacien et francique mosellan), flamand occidental, franco- 
provençal, langues d'oïl (franc-comtois, wallon, champenois, picard, normand, gallo, poitevin-saintongeais, lorrain, bourguignonmorvandiau), occitan ou langue d'oc (gascon, languedocien, provençal, auvergnat, limousin, vivaro-alpin), parlers liguriens.

Départements d'outre-mer:

Créoles guadeloupéen, guyanais, martiniquais, réunionnais, mahorais (shimaoré), malgache de Mayotte (shibushi)

Polynésie française:

Tahitien, marquisien, langue des Tuamotu, mangarévien, langues des Îles Australes

Wallis et Futuna:

Wallisien, futunien

Guyane:

Créole à base lexicale française ; créoles bushinenge (à base angloportugaise) : saramaka, aluku, njuka, paramaca ; langues amérindiennes : kali'na (ou galibi), wayana, palikur, arawak (ou lokono), wayampi, émerillon; hmong

Nouvelle-Calédonie (28 langues kanaks):

- Grande Terre : nyelâyu, kumak, caac, yuaga, jawe, nemi, fwâi, pije, pwaamei, pwapwâ, langue de Voh-Koné, cèmuhî, paicî, ajië, arhâ, arhö, 'ôrôê, neku, sîchë, tîrî, xârâcùù, xârâgùrè, drubéa, numèè

- Îles Loyauté : nengone, drehu, iaai, fagauvea

Source: d'après le rapport du Comité consultatif pour la promotion des langues régionales et de la pluralité linguistique interne, Redéfinir une politique publique en faveur des langues régionales et de la pluralité linguistique interne, juillet 2013, p. 92 (liste de langues établie par la Délégation générale à la langue française et aux langues de France). (République Française, 2015a).

Según lo expresado por la DGLFLF, no existe un listado exhaustivo de lenguas regionales o minoritarias a las que aplicar las disposiciones que figuran en la Car$t a$, una vez se ratifique. La relación de lenguas que aparece en el listado sería un bosquejo de lo que podría ser la selección final, eso sin olvidar que cada una de estas lenguas regionales o minoritarias tiene unas características particulares, que algunas son habladas por más de un millón de personas, y otras solo se utilizan de forma escrita y no se transmiten de padres a hijos (Leclerc, 2017).

Quizá el proceso de ratificación del que hablamos no parezca tan lejano, ya que, atendiendo a las constantes reclamaciones por parte de los habitantes de Córcega, Bretaña y el País Vasco francés, el expresidente Hollande solicitó con fecha 1 de junio de 2015, un proyecto de ley para modificar la Constitución, con el fin de poder finalmente ratificar la Carta (Rédaction de France Info, 2015). 
Sin embargo, el Consejo de Estado francés, que se reunió en julio de 2015 para tratar este tema, emitió un dictamen desfavorable con respecto a la modificación de la Constitución y la inserción del texto mencionado (Montvalon, 2015). Aun así, sigue presente en las agendas y se busca el camino para poder llegar a la ratificación.

En cualquier caso, Cerquiglini (Leclerc, 2017) hace hincapié en la vaguedad del conocimiento que se posee sobre el número y la realidad de las lenguas regionales o minoritarias en Francia, puesto que el último estudio en profundidad sobre el patrimonio lingüístico del país lo llevó a cabo el abad Grégoire en el siglo XVIII (17901792).

Una vez Francia ratifique la Carta europea de las lenguas regionales o minoritarias, estas lenguas minoritarias presentes en el territorio francés, quedarían protegidas y se velaría por su preservación, hecho de vital importancia, pues hasta el momento presente se han perdido gran cantidad de hablantes y, poco a poco, van cayendo en el olvido.

En este sentido, según la Organización de las Naciones Unidas para la Educación, la Ciencia y la Cultura (en adelante UNESCO), existen diferentes etiquetas para graduar la situación en la que se encuentran las lenguas en peligro de extinción. En su Atlas interactivo UNESCO de las lenguas del mundo en peligro (Moseley, 2010) la lista de lenguas en peligro asciende a 2.500 (de ellas, 230 han desaparecido desde el decenio de 1950).

Según la UNESCO, el nivel de vitalidad está directamente relacionado con la transmisión intergeneracional de la lengua (UNESCO, 2017). En la tabla siguiente se describen las características de cada uno de los niveles de vitalidad:

\begin{tabular}{|l|l|}
\hline \multicolumn{1}{|c|}{ NIVEL DE VITALIDAD } & \multicolumn{1}{c|}{$\begin{array}{c}\text { TRANSMISIÓN INTERGENERACIONAL } \\
\text { DE LA LENGUA }\end{array}$} \\
\hline A salvo (lenguas no incluidas en el Atlas) & $\begin{array}{l}\text { Todas las generaciones hablan la lengua y su transmisión } \\
\text { de una generación a otra es continua. }\end{array}$ \\
\hline Vulnerable & $\begin{array}{l}\text { La mayoría de los nińos hablan la lengua, pero su uso } \\
\text { puede estar restringido a determinados ámbitos (el hogar } \\
\text { familiar, por ejemplo). }\end{array}$ \\
\hline En peligro & $\begin{array}{l}\text { Los niños ya no la aprenden en sus familias como lengua } \\
\text { materna. }\end{array}$ \\
\hline Seriamente en peligro & $\begin{array}{l}\text { Solo los abuelos y las personas de las viejas generaciones } \\
\text { hablan la lengua. Los miembros de la generación parental, } \\
\text { si bien pueden comprenderla, no la hablan entre sí, ni } \\
\text { tampoco con sus hijos. }\end{array}$ \\
\hline En situación crítica & $\begin{array}{l}\text { Los únicos hablantes son los abuelos y las personas de las } \\
\text { viejas generaciones, pero solo usan la lengua parcialmente y } \\
\text { con escasa frecuencia. }\end{array}$ \\
\hline Extinta & $\begin{array}{l}\text { No quedan hablantes. (El Atlas contiene las referencias de } \\
\text { las lenguas extintas desde el ańo 1950.) }\end{array}$ \\
\hline
\end{tabular}


Tabla 2. Clasificación de los niveles de vitalidad de las lenguas según la UNESCO

En la actualidad, en Francia hay 26 lenguas en peligro. El picardo, por ejemplo, está marcado como lengua gravemente en peligro y, como dialecto del picardo, el ch'timi también ve cómo su número de hablantes se reduce poco a poco. Hagège (2000: 361) alerta sobre la importancia de concienciarse del problema de la desaparición de lenguas, del que quizás sólo los lingüistas alcanzan a ver la gravedad. Señala, asimismo, que esta situación se ve agravada por el hecho de que normalmente la prensa, la radio y la televisión emplean la lengua principal como medio de difusión, lo que va en detrimento de las lenguas minoritarias.

Cabe destacar, además, que el ch'timi varía ligeramente de una zona a otra, es decir, existen pequeñas diferencias entre el hablado por un habitante de Roubaix y por otro de Boulonnais (Dawson, 2011: 16), circunstancia esta que hace que no goce de cohesión formal. Quizá propuestas de unificación dentro de un mismo dialecto, como la de Feller-Carton, pensadas con el fin de dar cohesión a las diferentes variantes que utilizan los hablantes, ayudarían a que determinados dialectos sobrevivieran (Briales, 2018). La clave, en determinados casos, sería, por tanto, unificar para mantener la diversidad.

\section{Conclusiones}

La Francofonía, si nos asomamos al panorama lingüístico actual, está ligada, por un lado, a la heterogeneidad, a la pluralidad. Se trata de una diversidad entendida desde un prisma mundial: la variedad del francés utilizada en cada uno de los países en los que se habla; y desde una perspectiva nacional: la protección de los hablantes de lenguas minoritarias en el territorio francés, a través de los mecanismos que se han articulado en organizaciones internacionales. Es imprescindible cuidar la diversidad para mantener la riqueza. Por otro lado, la Francofonía se erige como un solo ente, siendo el francés el nexo de unión entre países lejanos y diversos, el punto en común que anima a trabajar juntos.

Ambas vertientes son reales y válidas, pero al igual que se respeta y se asumen las variedades del francés presentes en los cinco continentes, sería necesario trabajar en la unificación formal dentro de cada una de las lenguas minoritarias presentes en el territorio francés, ya que, en este caso, las diferencias pueden acarrear la desaparición y, con ello, se mermaría la riqueza lingüística existente ${ }^{3}$.

\footnotetext{
${ }^{3}$ La investigación realizada para el presente estudio forma parte de la tesis doctoral de la autora (Briales, 2015), defendida en la Universidad Pablo de Olavide el 21 de diciembre de 2015.
} 


\section{REFERENCIAS BIBLIOGRÁFICAS}

BARBIÈRE, Cécile (2014): «La France relance la ratification de la Charte européenne des langues regionales». Euractive [Consulta en línea: http://www.euractiv.fr/culture/lafrance-relance-la-ratificatio-news-533088; 14/11/2018].

BARTOlOnE, Claude (2014): Proposition de Loi Constitutionnelle adoptée par l'Assemblée Nationale, autorisant la ratification de la Charte européenne des langues régionales ou minoritaires ( $n^{\circ}$ 320). Session Ordinaire de 2013-2014. París, Sénat [Consulta en línea: http://www.senat.fr/leg/ppl13-320.pdf; 14/11/2018].

BECK Baptiste, Richard MARCOUX, Laurent RICHARD y Alexandre WOLFF (2018): Estimation des populations francophones dans le monde en 2018. Sources et démarches méthodologiques. Québec, Université Laval [Consulta en línea: http://www.odsef.fss.ulaval.ca/sites/odsef.fss.ulaval.ca/files/odsef-lfdm-2018.pdf; 07/11/2018].

BRIALES, Isabel (2015): La transferencia socio-cultural en Bienvenue Chez les Ch'tis. Tesis doctoral dirigida por la Dra. Pilar Rodríguez Reina. Sevilla, Universidad Pablo de Olavide.

BRIALES, Isabel (2018): «Diccionarios y lenguas minoritarias: el caso del ch'timi». Tonos digital, 34 [Consulta en línea: http://www.tonosdigital.es/ojs/index.php/tonos/article/view/1882/971; 14/11/2018].

CALVET, Louis-Jean (1999): La guerre des langues et les politiques linguistiques. París, Hachette Littératures.

CALVET, Louis-Jean (2007): «Approche sociolinguistique de l'avenir du français dans le monde». Hérodote, 126 (3), 153-160 [Consulta en línea: DOI: 10.3917/her.126.0153; $14 / 11 / 2018]$.

CONSEIL CONSTITUTIONNEL DE LA RÉPUblique FranÇAiSe (2018): Texte intégral de la Constitution du 4 octobre 1958 en vigueur. À jour de la révision constitutionnelle du 23 juillet 2008. París, Conseil Constitutionnel [Consulta en línea: http://www.conseilconstitutionnel.fr/conseil-constitutionnel/francais/la-constitution/la-constitution-du4-octobre-1958/texte-integral-de-la-constitution-du-4-octobre-1958-en-vigueur.5074.html\#titre1; 09/11/2018].

CONSEIL DE L'EUROPE (1992): Charte européenne des langues régionales ou minoritaires. Estrasburgo, Conseil de l'Europe [Consulta en línea: https://www.coe.int/fr/web/european-charter-regional-or-minority-languages/text-of-the-charter; 09/11/2018].

CONSEIL DE L'EUROPE (2018): État des signatures et ratifications du traité 148. Estrasburgo, Conseil de l'Europe [Consulta en línea: https://www.coe.int/fr/web/conventions/fulllist/-/conventions/treaty/148/signatures?p_auth=qRYvXbq3; 08/11/2018].

DAWSON, Alain (2011): Le ch'timi de poche. Parler du nord et du Pas-de-Calais. Chennevières sur Marne, Assimil.

GILDER, Alfred (1993): Et si l'on parlait français? París, Le Cherche Midi éditeur.

Gobierno de España (2015): Consejo de Europa. Madris, Ministerio de Asuntos Exteriores y Cooperación [Consulta en línea: http://www.exteriores.gob.es/PORTAL/ES/- 
POLITICAEXTERIORCOOPERACION/CONSEJODEEUROPA/Paginas/Inicio.aspx; 09/11/2018].

HAGĖGE, Claude (1987): Le français et les siècles. París, Éditions Odile Jacob.

HAGÈGE, Claude (2000): Halte à la mort des langues. París, Éditions Odile Jacob.

JACOT, Martine (2014): «Où en est la Francophonie ?». Le Monde, 30 novembre [Consulta en línea: https://www.lemonde.fr/afrique/visuel_interactif/2014/11/30/ou-en-est-lafrancophonie_4528533_3212.html; 14/11/2018].

LECLERC, Jacques (2017): «Rapport de Bernard Cerquiglini sur les langues de la France, Avril 1999. Rapport au ministre de l'Éducation Nationale, de la Recherche et de la Technologie, et à la ministre de la Culture et de la Communication», in L'aménagement linguistique dans le monde [Consulta en línea: http://www.axl.cefan.ulaval.ca/francophonie/Rapport-Cerquiglini-1999.htm; 02/11/2018].

MONTVAlON, Jean-Baptiste de (2015): «Nouvel obstacle à la ratification de la Charte des langues régionales». Le Monde, 1 de agosto [Consulta en línea: https://www.lemonde.fr/societe/article/2015/08/01/les-langues-regionales-bientot-reconnues-parla-constitution_4707451_3224.html; 14/11/2018].

MOSELEY, Christopher [ed.] (2010): Atlas de las lenguas del mundo en peligro. París, Ediciones UNESCO. [Consulta en línea: http://www.unesco.org/culture/languages-atlas/es/atlasmap.html; 09/11/2018].

ORGANISATION INTERNATIONALE DE LA FRANCOPHONIE (2013): Estimation des francophones [Consulta en línea: http://observatoire.francophonie.org/qui-parle-francaisdans-le-monde; 27/08/2015].

ORGANISATION INTERNATIONALE DE LA FRANCOPHONIE (2018a): Estimation des francophones [Consulta en línea: http://observatoire.francophonie.org/qui-parle-francaisdans-le-monde; 10/11/2018].

ORGANISATION INTERNATIONALE DE LA FRANCOPHONIE (2018b): L'Organisation Internationale de la Francophonie [Consulta en línea: https://www.francophonie.org/LOrganisation-internationale-de-la-Francophonie-42707.html; 10/11/2018].

ORGANISATION INTERNATIONALE DE LA FRANCOPHONIE (2018c): Diversité linguistique. [Consulta en línea: http://observatoire.francophonie.org/diversite-linguistique; $10 / 11 / 2018]$.

ORGANIZACIÓN DE LAS NACIONES UNIDAS PARA LA EDUCACIÓN, LA CIENCIA Y LA CULTURA (2017): Atlas UNESCO de las lenguas del mundo en peligro [Consulta en línea: http://www.unesco.org/new/es/culture/themes/endangered-languages/atlas-oflanguages-in-danger/; 14/11/2018].

RÉDACTION DE FRANCE INFO (2015): «Charte des langues régionales: Hollande lance le processus de ratification». France Info [Consulta en línea: http://www.franceinfo.fr/actu/politique/article/charte-des-langues-regionales-hollande-lance-leprocessus-de-ratification-688186; 09/11/2018]. 
RÉPUBLIQUE FRANÇAISE (2018a): Langue française et langues de France. París, Ministère de la Culture [Consulta en línea: http://www.culturecommunication.gouv.fr/Politiquesministerielles/Langue-francaise-et-langues-de-France/; 10/11/2018].

RÉPUBLIQUE FRANÇAISE (2015b): «Droit au français», in Langue française et langues de France. París, Ministère de la Culture [Consulta en línea: http://www.culturecommunication.gouv.fr/Politiques-ministerielles/Langue-francaise-et-languesde-France/Politiques-de-la-langue/Droit-au-francais; 09/11/2018].

URVOAS, Jean-Jacques (2014): «Rapport fait au nom de la Commission des Lois Constitutionnelles, de la Législation et de l'Administration Générale de la République, sur la proposition de Loi Constitutionnelle $\left(\mathrm{n}^{\circ} 1618\right)$ visant à ratifier la Charte européenne des langues régionales ou minoritaires. (Informe Núm. 1.703)». París, Asamblée Nationale [Consulta en línea: http://www.assemblee-nationale.fr/14/rapports/r1703.asp; 09/11/2018].

WOLFF, Alexandre (2010): La langue française dans le monde 2010. París, Éditions Nathan.

Para citar este artículo / Pour citer cet article :

BRIALES BELLÓN, Isabel (2019) : «Salvaguarda de la lengua francesa en el mundo y de las lenguas minoritarias en Francia: políticas e instituciones». Çédille, revista de estudios franceses, 16, $205-$ 225. DOI: https://doi.org/10.25145/j.cedille.2019.17.16.15. 